\title{
Cholangiopathy in critically ill patients surviving beyond the intensive care period: a multicentre survey in liver units
}

\author{
L. Laurent ${ }^{1}$, C. Lemaitre ${ }^{2}$, A. Minello ${ }^{3}$, A. Plessier ${ }^{1}$, G. Lamblin ${ }^{4}$, A. Poujol-Robert ${ }^{5}$, \\ A. Gervais-Hasenknopf ${ }^{6}$, E.-A. Pariente ${ }^{7}$, P. Belenotti ${ }^{8}$, N. Mostefa-Kara ${ }^{9}$, P. Sogni ${ }^{5}$, M. Legrand ${ }^{5}$, \\ J.-M. Cournac ${ }^{10}$, F. Tamion ${ }^{2}$, G. Savoye ${ }^{2}$, P. Bedossa ${ }^{1,5}$, D.-C. Valla ${ }^{1,5}$, V. Vilgrain ${ }^{1}$, O. Goria ${ }^{2}$
}

Summary

Background: The outcome of cholangiopathy developing in intensive care unit (ICU) is not known in patients surviving their ICU stay.

Aim: To perform a survey in liver units, in order to clarify the course of cholangio-pathy after surviving ICU stay. Methods: The files of the liver units affiliated to the French network for vascular liver disease were screened for cases of ICU cholangiopathy developing in patients with normal liver function tests on ICU admission, and no prior history of liver dis-ease.

Results: Between 2005 and 2015, 16 cases were retrieved. Extensive burns were the cause for admission to ICU in 11 patients. Serum alkaline phosphatase levels increased from day 11 (2-46) to a peak of 15 (4-32) 9 ULN on day 81 (12-511). Magnetic resonance cholangiography showed irregularities or frank stenosis of the intrahepatic ducts, and proximal extrahepatic ducts contrasting with a normal aspect of the distal common bile duct. Follow-up duration was 20.6 (4.7-71.8) months. Three patients were lost to follow-up; 2 patients died from liver failure and no patient was transplanted. One patient had worsening strictures of the intrahepatic bile ducts with jaundice. Nine patients had persistent but minor strictures of the intrahepatic bile ducts on MR cholangiography, and persistent cholestasis without jaundice. One patient had normal liver function tests.

Conclusions: In patients surviving their ICU stay, ICU cholangiopathy is not uni-formly fatal in the short term or clinically symptomatic in the medium term. Preser-vation of the distal common bile duct appears to be a finding differentiating ICU cholangiopathy from other diffuse cholangiopathies. 


\section{1 | INTRODUCTION}

Cholangiopathy in critically ill patients, so-called intensive care unit (ICU) cholangiopathy, is a recently described ischaemic-like form of cholangiopathy. The first case was reported in 2001 by Scheppach et al. ${ }^{1}$ Reports of isolated cases or small series have mostly originated from intensive care units, in patients with polytrauma, infection, burn, major surgery or respiratory failure. ${ }^{1-3}$ ICU cholangiopathy is characterised by the formation of biliary casts at an early stage. Subsequent changes include the progressive destruction of intrahepatic bile ducts with multifocal strictures and dilatations. ${ }^{2-4}$ Rapid progression to acute liver failure has been reported. ${ }^{3}$ Average survival time, without liver transplantation, is only 13 months. ${ }^{5-7}$ Pathogenesis has been poorly investigated. ${ }^{3,8}$ Currently available clinical surveys have been conducted in ICU and are mostly based on endoscopic retrograde cholangiopancreatography (ERCP) findings, providing little information on the sequelae of the disease in patients surviving intensive care and thus referred to liver units. Therefore, the aims of this present survey were to describe the clinical, laboratory and imaging (MRI) features, as well as the characteristics and the outcome of patients with ICU cholangiopathy referred to liver units.

\section{2 | PATIENTS AND METHODS}

Cases were retrospectively identified through a survey conducted in French liver units affiliated with two French reference centres for rare diseases: the vascular liver diseases centre at Beaujon University Hospital, in the greater Paris area; and the inflammatory biliary diseases centre at Saint Antoine University Hospital, Paris, France. Diagnosis of cholangiopathy in critically ill patients was based on the following three criteria: (1) normal liver function tests before admission to ICU; (2) development of persistent cholestasis after admission to ICU; and (3) irregularities of the intrahepatic ducts on magnetic resonance cholangiopancreatography. Patients were excluded from the study if they had a history of liver disease or inflammatory bowel disease prior to ICU admission; and if trauma had induced injury to splanchnic vessels. Cholestasis was defined as an increase in alkaline phosphatase (AP) activity more than twice the upper limit of normal values (ULN). Clinical, laboratory, histopathological and imaging data were collected using a structured questionnaire, on the day of admission, during ICU stay and during post-ICU follow-up. ICU records were analysed for cause of admission, use of mechanical ventilation, vasopressors, transfusions, systemic inflammatory response syndrome (SIRS), sepsis and antibiotic therapy.

One expert abdominal radiologist (V.V.) reviewed all MRI while blinded to clinical and laboratory parameters. The following sequences were analysed: (1) T1-weighted sequence, axial, (2) fatsuppressed T2-weighted sequence, axial, (3) single-shot T2-weighted sequence, axial HR, (4) diffusion-weighted sequence, (5) 2D and 3D MR cholangiography, (6) contrast-enhanced sequences using extracellular contrast agents. All sequences including native and 3D MIP (Maximal intensity projection) reconstructions were analysed on a workstation. For each patient, the following items were studied using a standardised scale: intrahepatic biliary duct abnormalities, main bile duct abnormalities, abnormalities of the hepatic parenchyma, parenchymal enhancement after injection, enhancement of bile duct walls, hepatic arterial abnormalities, signs of portal hypertension (splenomegaly, portosystemic collaterals) and morphological changes of the liver.

Treatment modalities during follow-up were collected including: endoscopic treatment, ursodeoxycholic acid therapy and liver transplantation.

\section{1 | Statistical analysis}

All results are expressed as median and range. Categorical data are expressed as frequency and percentage.

\section{\begin{tabular}{l|l}
3 & RESULTS
\end{tabular}}

We identified 19 patients with cholangiopathy, 3 of whom were excluded from analysis because of mesenteric deinsertion in one, hepatic artery dissection and inability to review the images in the others.

Finally, 16 patients were included (11 male, 5 female). The median age at diagnosis was 52 years (16-83). Seven patients had at least one cardiovascular risk factors on admission (4 patients were current smokers, 3 had hypercholesterolemia, 1 arterial hypertension, 1 diabetes). None of the patients reported alcohol misuse. One patient had received chemotherapy for B-cell lymphoma. One patient had a documented MDR3 (ABCB4) mutation. As shown in Table 1, extensive burns and polytrauma were the predominant reasons for ICU admission. All patients presented with hemodynamic instability on admission. Eleven patients required noradrenaline treatment. The median duration of noradrenaline therapy was 6 days (1-42). Fifteen patients required transfusions. The median number of red blood cell units (RBC) transfused was 26 (range: 2-94). Fifteen patients had mechanical ventilation for a median duration of 51 days (range: 20-136).

The median duration of ICU stay was 72 days (range: 1-172). As presented in Table 2, the increase in serum AP levels started after a median time of 11 days, reaching a peak of 15 on average and at the upper limit of normal values (ULN), a median of 3 months after admission. However, there were marked variations in time course according to patients. During hospitalisation, all patients presented a systemic inflammatory response syndrome (SIRS) and at least one bacterial infection. The most frequent infections were septicaemia in 13 patients, pneumonia in 13 and skin infection in 12. Infections were caused by various species (Pseudomonas aeruginosa, enterobacter cloacae, streptococcus pneumoniae, staphylococcus aureus etc.). Bacterial examination of the bile was not performed. 
TABLE 1 Length of stay, use of vasoactive agents, mechanical ventilation and transfusion according to the cause of admission to ICU

\begin{tabular}{|c|c|c|c|c|c|c|c|c|}
\hline \multirow[b]{2}{*}{ Causes } & \multirow[b]{2}{*}{ Patients } & \multicolumn{2}{|c|}{ Vasoactive agents } & \multicolumn{2}{|c|}{ Mechanical ventilation } & \multicolumn{2}{|l|}{ Transfusion } & \multirow[b]{2}{*}{$\begin{array}{l}\text { Length of ICU } \\
\text { stay-days } \\
\text { median (range) }\end{array}$} \\
\hline & & $\begin{array}{l}\text { Patients, } \\
\text { n (\%) }\end{array}$ & $\begin{array}{l}\text { Days, median } \\
\text { (range) }\end{array}$ & $\begin{array}{l}\text { Patients, } \\
\text { n (\%) }\end{array}$ & $\begin{array}{l}\text { Days, median } \\
\text { (range) }\end{array}$ & $\begin{array}{l}\text { Patients, } \\
\text { n (\%) }\end{array}$ & $\begin{array}{l}\text { RBC transfusion } \\
\text { unit per patient } \\
\text { (median, range) }\end{array}$ & \\
\hline Burns & 11 & 8 (73\%) & $5(1-42)$ & $11(100 \%)$ & $48(2-58)$ & 11 (100\%) & $26(2-94)$ & $76(35-172)$ \\
\hline Polytrauma & 3 & $3(100 \%)$ & $23(6-32)$ & 3 (100\%) & $51(37-52)$ & $3(100 \%)$ & $47(7-48)$ & $56(40-58)$ \\
\hline Infection & 1 & 0 & & 0 & 69 & 0 & & 1 \\
\hline ARDS & 1 & 1 & 3 & 1 & $69(1-172)$ & 1 & 16 & 69 \\
\hline
\end{tabular}

RBC, red blood cell units; ARDS, acute respiratory distress syndrome.

TAB LE 2 Liver function tests

$\begin{array}{llll} & \begin{array}{l}\text { Time to first } \\ \text { increase (days), } \\ \text { (median, range) }\end{array} & \begin{array}{l}\text { Time to peak } \\ \text { level (days), } \\ \text { (median, range) }\end{array} & \begin{array}{l}\text { Peak level } \\ \text { (UNL) (median, } \\ \text { range) }\end{array} \\ \text { AP } & 11[2-46] & 81[12-511] & 15[4-32] \\ \text { gammaGT } & 9[4-19] & 84[26-476] & 28[18-119] \\ \text { Total Bilirubin } & 26[10-84] & 74[17-426] & 15[3-33] \\ \text { ALAT } & - & 43[20-84] & 7[6-16]\end{array}$

UNL, upper limit of normal values; AP, alkaline phosphatase.

All patients had at least one MRI examination of the liver including MR cholangiography (range, 1-3). Median delay between ICU admission and first MRI was 4 months (0.5-20.0). At initial MRI, 11 of 16 patients had endoluminal defects of the bile ducts corresponding to biliary casts, which was confirmed by ERCP in three patients (median: 3.5 months [0.5-7.6]). Five patients had biliary leakage with hyperintense collections on T2-weighted MR images communicating with bile ducts, suggestive of bilomas (median: 3.8 months [0.5-6.5]) (Figure 1). All but three patients had multiple strictures of the intrahepatic bile ducts. Strictures were $>1 \mathrm{~cm}$ in length at the level of the right, left or upper common hepatic ducts in 11 patients. These long strictures were associated with shorter strictures of peripheral or peripheral and central intrahepatic bile ducts. Fifteen patients had thickened and enhancing intrahepatic bile duct walls (Figure 2). The common bile duct (CBD) was not dilated and there was no abnormal thickening of the CBD wall. Liver morphology was normal in all but two patients at the first MRI (hypertrophy of the caudate lobe and segment IV for one and hypertrophy of segment IV for the other). The hepatic artery and its main branches were patent in all patients on contrast-enhanced MR images.

A second MRI was performed in eight patients. Median delay between admission to ICU and second MRI was 15.6 months (5.227.3). Two patients developed diffuse strictures of the intrahepatic bile ducts and one had a rarefaction of the intrahepatic bile ducts. Abnormal portal veins were observed in two other patients: splenomegaly, portosystemic collaterals with thrombosis of segmental portal branches in one; and thrombosis of the left portal branch without features of portal hypertension in the other. At an early stage, most patients had defects in the intrahepatic biliary tree corresponding to biliary casts and biliary leakages. At a later stage, MR cholangiography showed long hilar strictures, whereas the distal common bile duct appeared normal.

Liver biopsy, performed in 6 patients, 130 (45-668) days after admission to ICU, showed significant alterations of interlobular bile duct epithelium such as nuclear pseudostratification, cytoplasmic clarification/vacuolisation of biliary epithelial cells or cell desquamation in the bile duct lumen. Arterioles and venules were present and patent in the portal tracts. Portal and periportal mild mixed inflammatory infiltrate was also described. Ductular reaction and bile duct infarcts close to the portal tracts were observed in two patients. No periportal or lobular necroinflammatory lesions were seen. Marked centrilobular cholestasis was detected. Two years (range 1.8-3.1) after admission, we observed portal and periportal fibrosis in 4 patients and biliary cirrhosis in 2 patients.

Three patients were lost to follow-up after a median delay of 5.3 months (4.9-30.9), leaving 13 patients for outcome analysis. Median follow-up was 20.6 months (range: 4.7-71.8) (Table 3). All patients received ursodeoxycholic acid (UDCA) $(10-15 \mathrm{mg} / \mathrm{kg} / \mathrm{d}$ ). ERCP with endoscopic sphincterotomy of the papilla was performed in 4 patients with persistent jaundice, 4.1 months (2.6-5.1 months) after admission to ICU. The material removed was not analysed. Two patients had persistent severe cholestasis with jaundice and severe cholangiopathy. They died from hepatic failure. The first patient died 20.5 months after admission, being considered too sick for liver transplantation. The second patient, a 79-year-old male, died 71.8 months after initial admission for hepatic encephalopathy secondary to biliary cirrhosis. No patient was transplanted. One patient had worsening strictures of the intrahepatic bile ducts with jaundice without complication. Nine patients had persistent but minor strictures of the intrahepatic bile ducts on MR cholangiography, and persistent cholestasis without jaundice. One patient had normal liver function tests.

\section{4 | DISCUSSION}

We report here, a retrospective multicentre study on a cohort of 16 patients surviving a critical illness in ICU and affected with cholangiopathy, as diagnosed subsequently by hepatologists. Although the small number of patients and the heterogeneous management, all related to the rarity of the disease, constitute limitations to the 

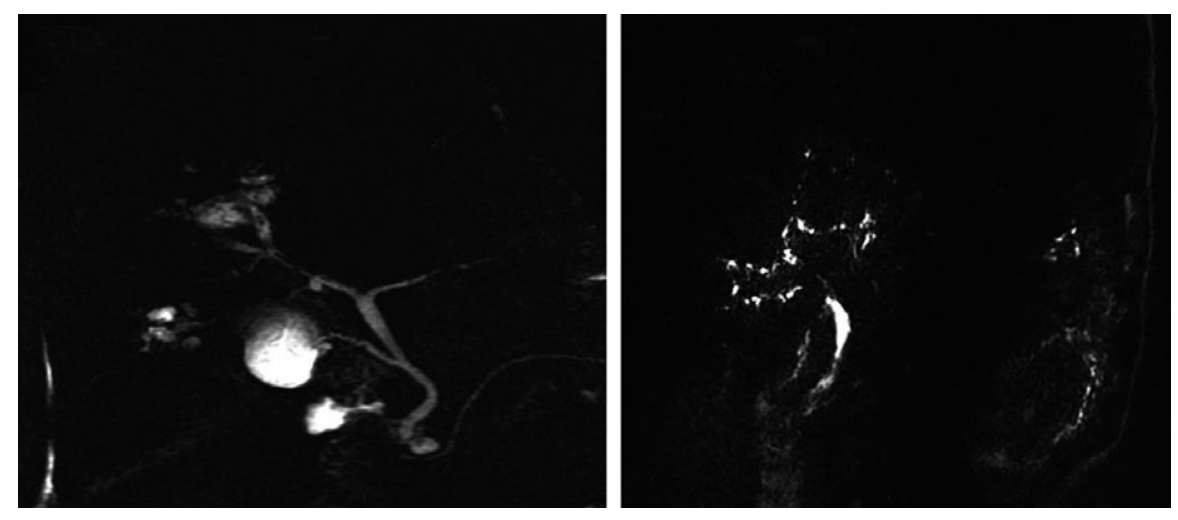

FIGURE 1 MR cholangiography at an early stage. Defects corresponding to casts and extrabiliary collection of bile corresponding to bilomas (Left: At 2 mo after diagnosis. Right: At 7 mo after diagnosis)
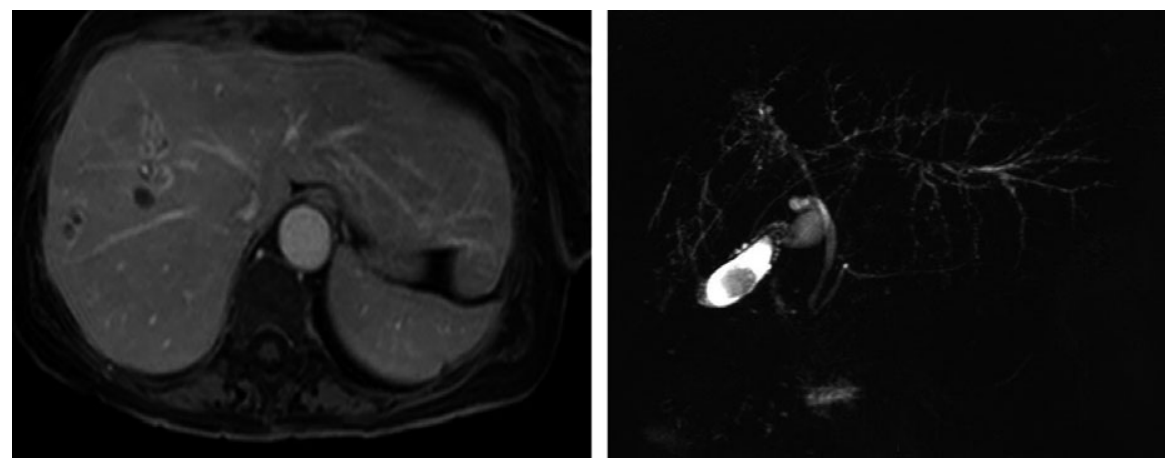

FIGURE 2 Contrast-enhanced MR obtained at the portal venous phase and magnetic resonance cholangiopancreatography (At 4 mo after diagnosis): Rail thickening corresponding to thick and enhancing bile duct wall (left). Series of strictures and dilatations (right)

interpretation of our study; novel and solid findings include a specific MR cholangiographic aspect, a better outcome than previously reported, and a preponderance of burns as the cause of admission to ICU.

A major strength of the present study was the availability of both initial MRI and MR cholangiography imaging, as well as followup MRI in half of the cohort. In previously reported surveys with two exceptions, ${ }^{9,10}$ imaging studies were limited to ERCP. Conversely, in the current study we observed different imaging patterns according to the delay of the disease. At an early stage (median: 3.5 months), imaging showed luminal alterations of the intrahepatic biliary tree corresponding to biliary casts and biliary leakages in most patients. At a later stage, MR cholangiography showed long hilar strictures, whereas the distal common bile duct appeared normal.

The findings of our multicentre survey in liver units independently validate previously reported observations in ICU based surveys; notably a mean age of 50 years, a male predominance, and a rapid development of cholestasis after admission to ICU in patients who had no prior history of liver disease and normal liver function tests before admission. ${ }^{2-4}$

Cholestatic liver function tests are very common in ICU, due to sepsis, whatever the origin, or due to parenteral feeding or are druginduced, however, the vast majority of such cases do not have demonstrable cholangiopathy. MRI is indicated when cholestasis persists after recovery from the ICU stay and with particularly elevated levels of alkaline phosphatase or when clinical features suggest biliary complications such as cholangitis.

A second strength of this present survey was the availability of long-term follow-up data in a specific subset of patients having survived ICU stay, a population of major interest for hepatologists. The outcome of cholangiopathy seems better than that reported in the literature, as described in Table 4. Indeed, most published cases have been reported by intensivists, who diagnosed severe cholangiopathy, cholangitis, liver abcesses and poor outcome without transplantation during ICU stay. Kulaksiz et al in 2008 estimated a median survival time of 13 months without liver transplantation. ${ }^{5}$ After a median follow-up of 20.6 months, 11 of our patients (68.8\%) were alive without liver transplantation, including 9 with cholestasis but without jaundice. However, two patients died due to acute liver failure, pointing to an extreme variability in the expression and outcome of the disease. These two patients presented to ICU with early severe disease as described in previously reported surveys. The design of our study does not allow for the identification of prognostic factors for the severity of bile duct disease.

In our study, most patients had burns and trauma (11 of 16 patients and 3 of 16 patients respectively). It must be emphasised that cases were collected on the basis of those patients who survived ICU stay, that is, patients in very good condition at the time of their critical illness. This in turn explains the predominance of accidents, (including burns and trauma) in previously healthy people as a 
TABLE 3 Follow-up (months)

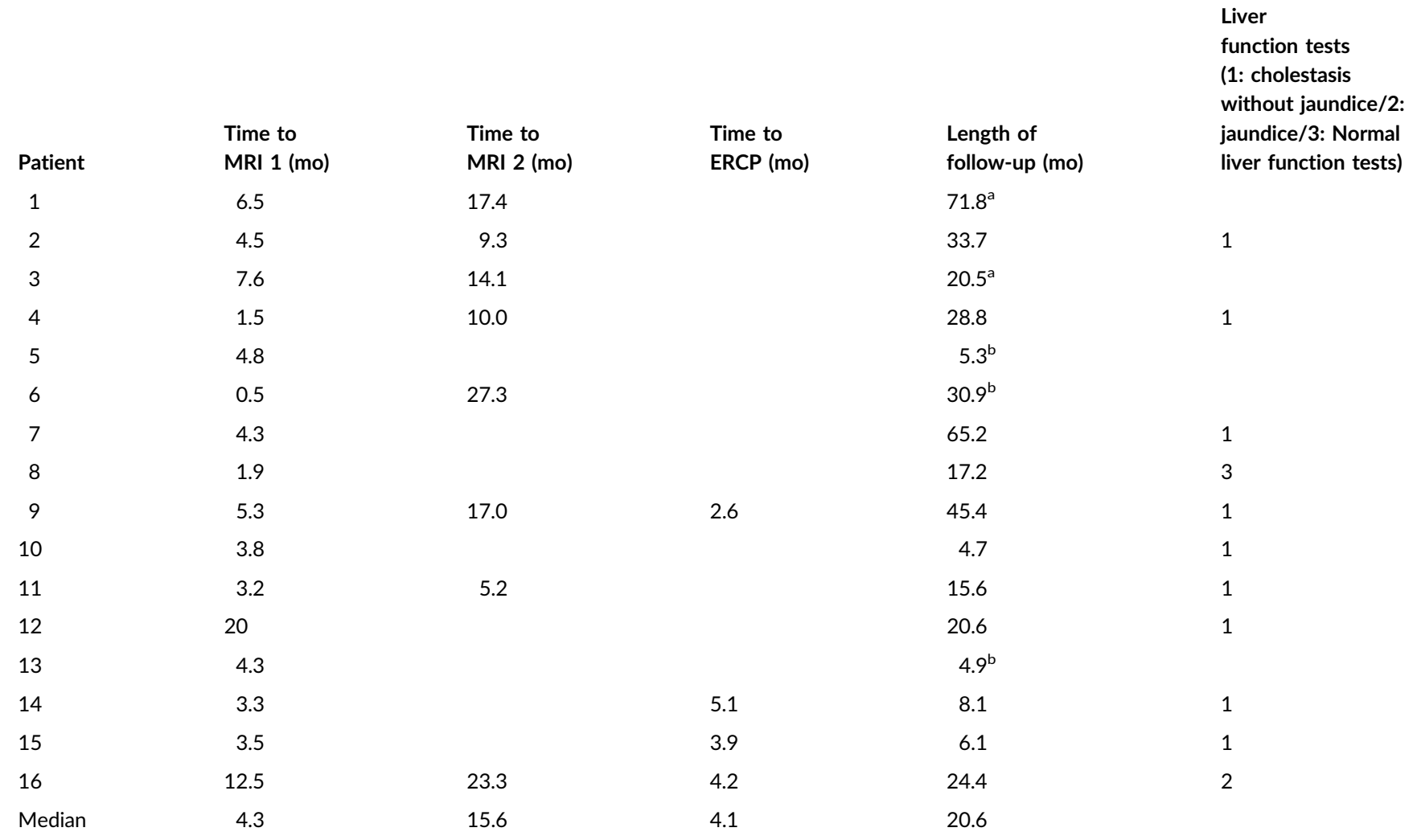

MRI, magnetic resonance imaging; ERCP, endoscopic retrograde cholangiopancreatography.

${ }^{a}$ Death.

bost to follow-up.

TABLE 4 Comparison between data in the literature

\begin{tabular}{|c|c|c|c|c|c|}
\hline Author & $\begin{array}{l}\text { Trauma } \\
\text { (n) }\end{array}$ & $\begin{array}{l}\text { Burns } \\
\text { (n) }\end{array}$ & Follow-up (mo) & $\begin{array}{l}\text { Liver } \\
\text { transplantation } \\
\text { (n) }\end{array}$ & $\begin{array}{l}\text { Death due } \\
\text { to hepatic } \\
\text { failure }(n)\end{array}$ \\
\hline Jaeger $^{6}$ & $5 / 10$ & $0 / 10$ & $21.0(3-54)$ & $1 / 10$ & $0 / 10$ \\
\hline Gelbmann $^{3}$ & $7 / 26$ & $0 / 26$ & $(4-49)$ & $7 / 26$ & $7 / 26$ \\
\hline Kulaksiz $^{5}$ & $5 / 29$ & $5 / 29$ & $21.0(1-123)$ & $7 / 29$ & $18 / 29$ \\
\hline Esposito $^{18}$ & $1 / 10$ & $1 / 10$ & $14.4(2-92)$ & $0 / 10$ & $5 / 10$ \\
\hline $\begin{array}{l}\text { Our study, } \\
2017\end{array}$ & $3 / 16$ & $11 / 16$ & $20.6(4.7-71.8)$ & $0 / 16$ & $2 / 16$ \\
\hline
\end{tabular}

cause for admission to ICU. Furthermore, severe burns require prolonged care and thus allowed for prolonged surveillance. Moreover, in ICU the death rate for patients with burns is lower than for all other pathologies ( $25 \%$ vs $50-70 \%$ ), probably because these patients are younger, with fewer comorbidities and a different pathophysiology for critical conditions. ${ }^{11,12}$

The pathophysiology of ICU cholangiopathy in critically ill patients is likely complex and multifactorial, because it appears to be rare and to affect patients with diverse underlying conditions. Unlike the hepatic parenchyma, the intrahepatic biliary tree is exclusively supplied by the hepatic arterial branches, via the peribiliary vascular plexus. ${ }^{13}$ Ischaemia due to hypovolaemia and hypoxaemia could be the first hit in cholangitis in critically ill patients, vasoconstrictive therapy might constitute an aggravating factor. ${ }^{3,14}$ In our cohort, MR imaging findings showed no damage of the distal common bile duct in any of our 16 patients, which confirmed the findings of Benninger et al in 5 patients using ERCP. ${ }^{10}$ Unlike the intrahepatic biliary tree, which is exclusively supplied by the hepatic arterial branches, the common bile duct is also supplied by gastroduodenal arterial branches. ${ }^{15}$ All these elements argue for the involvement of hepatic arterial microcirculation.

The clinical and imaging features of ICU cholangiopathy described here are very similar to those described in cases of ischaemic cholangiopathy after liver transplantation including bile cast formation or bilomas and the aspect of primary sclerosing cholangitis. Several factors likely contribute to arterial ischaemia at liver 
transplantation ${ }^{16,17}$ : preservation injury to the peribiliary plexus, hepatic artery thrombosis, the suppression of arterial collaterals by explantation, cytomegalovirus infection and rejection. The resemblance of ICU cholangiopathy with cholangiopathy after liver transplantation with a patent hepatic artery is even more striking: indeed, the strictures also predominantly affect the middle third of the common bile duct, with relative preservation of the distal common bile duct. These similarities reinforce the ischaemic hypothesis of the ICU cholangiopathy.

\section{5 | CONCLUSIONS}

MR cholangiography is an easy, non-invasive, complete imaging method in transportable patients for the diagnosis of ICU cholangiopathy in patients surviving beyond the intensive care period. The preservation of the distal common bile duct is suggestive of this diagnosis in this context. The spectrum of severity of the disease is probably wider than previously suspected: fewer severe features and better outcomes of the disease have been observed, after ICU stay, particularly in patients with burns.

\section{ACKNOWLEDGEMENTS}

We are grateful to hepatologists from 18 liver centres in France belonging to 2 national networks (the rare vascular liver diseases network and the rare biliary diseases network) for help for recruitment of cases. All of them had answered but 8 had no case to propose. We are grateful to Nikki Sabourin-Gibbs (Rouen University Hospital) for help in editing the manuscript.

Declaration of personal and funding interests: None.

\section{AUTHORSHIP}

Guarantor of the article: LL.

Author contributions: LL, DCV, VV, OG: contributed to study conception and design. LL, OG: contributed to data acquisition. LL, DCV, VV, OG: contributed to data analysis and interpretation. LL, $\mathrm{CL}, \mathrm{AM}, \mathrm{AP}, \mathrm{GL}, \mathrm{APR}, \mathrm{AGH}, \mathrm{EAP}, \mathrm{PB}, \mathrm{NMK}, \mathrm{PS}, \mathrm{ML}, \mathrm{JMC}, \mathrm{FT}, \mathrm{GS}$ $\mathrm{PB}, \mathrm{DCV}, \mathrm{VV}, \mathrm{OG}$ : wrote the paper. LL, DCV, VV, OG : contributed to editing.

All authors approved the final version of the manuscript.

\section{ORCID}

L. Laurent iD http://orcid.org/0000-0003-0674-736X

\section{REFERENCES}

1. Scheppach W, Druge G, Wittenberg G, et al. Sclerosing cholangitis and liver cirrhosis after extrabiliary infections: report on three cases. Crit Care Med. 2001;29:438-441.
2. Lin $\mathrm{T}, \mathrm{Qu} \mathrm{K}, \mathrm{Xu} \mathrm{X}$, et al. Sclerosing cholangitis in critically ill patients: an important and easily ignored problem based on a German experience. Front Med. 2014;1:118-126.

3. Gelbmann CM, Rümmele $P$, Wimmer $M$, et al. Ischemic-like cholangiopathy with secondary sclerosing cholangitis in critically ill patients. Am J Gastroenterol. 2007;102:1221-1229.

4. Al-Benna S, Willert J, Steinau HU, et al. Secondary sclerosing cholangitis, following major burn injury. Burns. 2010;36:e106-e110.

5. Kulaksiz H, Heuberger D, Engler S, et al. A. Poor outcome in progressive sclerosing cholangitis after septic shock. Endoscopy. 2008;40:214-218.

6. Jaeger C, Mayer G, Henrich R, et al. Secondary sclerosing cholangitis after long-term treatment in an intensive care unit: clinical presentation, endoscopic findings, treatment, and follow-up. Endoscopy. 2006;38:730-734.

7. Gl Kirchner, Scherer MN, Obed A, et al. Outcome of patients with ischemic-like cholangiopathy with secondary sclerosing cholangitis after liver transplantation. Scand J Gastroenterol. 2011;46:471-478.

8. Ruemmele P, Hofstaedter F, Gelbmann CM. Secondary sclerosing cholangitis. Nat Rev Gastroenterol Hepatol. 2009;6:287-295.

9. Kwon ON, Cho SH, Park CK, et al. Biliary cast formation with sclerosing cholangitis in critically ill patient: case report and literature review. Korean J Radiol. 2012;13:358-362.

10. Benninger J, Grobholz R, Oeztuerk Y, et al. Sclerosing cholangitis following severe trauma: description of a remarkable disease entity with emphasis on possible pathophysiologic mechanisms. World $J$ Gastroenterol. 2005;11:4199-4205.

11. Pham TN, Kramer CB, Wang J, et al. Epidemiology and outcomes of older adults with burn injury: an analysis of the National Burn Repository. J Burn Care Res. 2009;30:30-36.

12. Higgins T, McGee WT, Steingrib JS, et al. Early indicators of prolonged intensive care unit stay: impact of illness severity, physician staffing, and pre-intensive care unit length of stay. Crit Care Med. 2003;31:45-51.

13. Deltenre P, Valla DC. Ischemic cholangiopathy. J Hepatol. 2006;44:806-817.

14. Weig T, Schubert MI, Gruener N, et al. Abdominal obesity and prolonged prone positioning increase risk of developing sclerosing cholangitis in critically ill patients with influenza A-associated ARDS. Eur J Med Res. 2012;22:17-30.

15. Kramer L. Mechanism and management of cholestasis in sepsis and post-operative care. Postgraduate EASL 2011.

16. Cameron AM, Busuttil RW. Ischemic cholangiopathy after liver transplantation. Hepatobiliary Pancreat Dis Int. 2005;4:495-501.

17. Pasher A, Neuhaus P. Bile duct complications after liver transplantation. Transplant Int. 2005;18:627-642.

18. Esposito I, Kubisova A, Stiehl A, et al. Secondary sclerosing cholangitis after intensive care unit treatment:clues to the histopathological differential diagnosis. Virchows Arch. 2008;453:339-345. 


\section{APPENDIX}

\section{AUTHORS' COMPLETE AFFILIATIONS}

Lucie Laurent: DHU Unity, Service de Pancréatologie, Hôpital Beaujon, AP-HP, Clichy, France. Caroline Lemaitre: Service d'Hépatologie et Gastroentérologie, CHU Rouen; INSERM U1073, Rouen, France. Anne Minello: Service d'Hépatologie, Gastroentérologie et Oncologie digestive, CHU Dijon; EPICAD LNC-UMR1231, Burgundy \& Franche Comté University, Dijon, France. Aurélie Plessier: DHU Unity, Service d'Hépatologie, Hôpital Beaujon, AP-HP, Clichy, France. Géraldine Lamblin: Service d'Hépatologie et Gastroentérologie, $\mathrm{CHU}$ Clermont-Ferrand, Clermont-Ferrand, France. Armelle Poujol-Robert: Service d'Hépatologie, Hôpital Saint-Antoine, AP-HP, Université Pierre et Marie Curie, Paris, France. Anne Gervais-Hasenknopf: Service d'Hépatologie et Gastroentérologie, Hôpital Louis Mourier, AP-HP, Colombes, France. Emile-Alexandre Pariente: Service d'Hépatologie et Gastroentérologie, Centre hospitalier de Pau, Pau, France. Pauline Belenotti: Service de Médecine Interne, CHU La Timone, Marseille, France. Nassim Mostefa-Kara: Service d'Hépatologie et
Gastroentérologie, Centre Hospitalier Intercommunal d'ElbeufLouviers-Val de Reuil, Elbeuf, France. Philippe Sogni: Service d'Hépatologie, Hôpital Cochin, AP-HP; Université Paris Descartes et INSERM U-1223, institut Pasteur, Paris, France. Matthieu Legrand: Service d'Anesthésie, Réanimation, Centre de traitement des brûlés, Hôpital Saint-Louis, AP-HP ; INSERM UMR-S942, Institut National de la Santé et de la Recherche Médicale (INSERM), Lariboisière hospital; Université Paris Diderot, F-75475, Paris, France. Jean-Marie Cournac: Service de Médecine Interne, Hopital d'Instruction des Armees Percy, Clamart, France. Fabienne Tamion: Service de Réanimation, CHU Rouen, Rouen, France. Guillaume Savoye: Service d'Hépatologie et Gastroentérologie, CHU Rouen, Rouen, France. Pierre Bedossa: DHU Unity, Service d'Anatomopathologie, Hôpital Beaujon, AP-HP, Clichy; CRI, UMR 1149, Université Paris-Diderot and INSERM, Paris, France. Dominique-Charles Valla: DHU Unity, Service d'Hépatologie, Hôpital Beaujon, AP-HP, Clichy; CRI, UMR 1149, Université Paris-Diderot and INSERM, Paris, France. Valérie Vilgrain: DHU Unity, Service de Radiologie, Hôpital Beaujon, AP-HP, Clichy, France. Odile Goria: Service d'Hépatologie et Gastroentérologie, CHU Rouen, Rouen, France 